EESTI NSV TEADUSTE AKADEEMIA TOIMETISED. XV KÖIDE FUUSIKA-MATEMAATIKA- JA TEHNIKATEADUSTE SEERIA. 1966, NR. 3

ИЗВЕСТИЯ АКАДЕМИИ НАУК ЭСТОНСКОН̈ ССР. ТОМ ХV

СЕРИЯ ФИЗИКО-МАТЕМАТНЧЕСКИХ И ТЕХНИЧЕСКИХ НАУК. 1966, № 3

Л. АННОЛА

\title{
АСИМПТОТИЧЕСКАЯ ТЕОРИЯ ДИНАМИКИ УПРУГИХ ПЛАСТИНОК ПРИ БОЛЬШИХ ПЕРЕМЕЩЕНИЯХ
}

В работе [1] исследовалось асимптотическое интегрирование уравнений геометрически нелинейной теории упругости в случаях, когда величины перемещений имеют порядки

$$
u_{\alpha .} \sim \varepsilon h \div \varepsilon^{2} h, \quad u_{3} \sim h \div \varepsilon h, \quad \varepsilon=h / L
$$

(здесь $h-$ полутолщина, $L-$ характерная ширина пластинки). Поведение пластинки при таких перемещениях описывается теорией Кармана, линейной теорней совместно c плоским напряженным состоянием или линейной теорией.

В настоящей работе асимптотическое интегрирование нелинейных уравнений теории упругости проводится в случае существенно больших величин перемещений при дробных порядках $\varepsilon$. Рассматриваются напряженные состояния с $u_{a}-h \div \varepsilon^{1 / 2} h$, $u_{3} \sim \varepsilon^{-1 / 2} h \div h$ и применяется такая же методика, как в работе [1]: уравнения движения и граничные условия выводятся из требования, чтобы вариация соответствующего функционала равнялась нулю с требуемой асимптотической точностью.

Оказывается, что перемещения указанных порядков определяются при помощи мембранной теории.

Перечень литературы по асимптотическому методу интегрирования и по различным нелинейным теориям пластинок приведен в работе [1].

\section{1. Асимптотическое разложение в нелинейной теории упругости}

Пусть геометрически нелинейная теория упругости в прямоугольных координатах $x_{i}(i=1,2,3)$ и во времени $t$ описывается компонентами тензора напряжений $\sigma_{i k}$, тензора деформаций $e_{i k}$ и вектора перемещений $u_{i}$.

Введем новые безразмерные координаты

$$
\xi_{\alpha}=\varepsilon \frac{x_{\alpha}}{h}, \quad \eta=\frac{x_{3}}{h}, \quad(\alpha, \beta, \gamma, \ldots=1,2)
$$

где $\varepsilon=h / L$ - малый параметр; $2 h$ - толщина пластинки; $L$ - характерный размер пластинки по координатам $x_{\alpha}$.

Пусть введены также безразмерные перемешения $v_{\alpha}$,

$$
u_{\alpha}=\varepsilon^{s} h \tau_{\alpha}, \quad u_{3}=\varepsilon^{m} h \omega .
$$


В новых переменных деформации в плоскости пластинки будут

$$
e_{\alpha, \beta}=\frac{1}{2} \varepsilon^{1+s}\left(v_{\alpha, \beta}+v_{\beta, \alpha}+\varepsilon^{1+2 m-s} \psi_{, \alpha} \mho_{, \beta}+\varepsilon^{1+s} v_{\gamma, \alpha} v_{\gamma, \beta}\right) \text {. }
$$

Здесь производные по $\xi_{\alpha}$ обозначены: $\frac{\partial}{\partial \xi_{\alpha}}(\quad)=(\quad), a$.

Поскольку $\sigma_{\alpha \beta} \sim \mu e_{\alpha}$, то для того, чтобы оставаться в рамках теории упругости, надо потребовать, чтобы $1+s>0$. Иначе было бы $\sigma_{\alpha \beta} \sim \mu$. Кроме того, в теориях, сохраняющих геометрическую нелинейность выражения (1.3), надо взять $1+2 m-s \leqslant 0$. Если считать $s$ целым числом и рассматривать только такие варианты, когда в выражениях (1.3) сохраняются в первом приближении линейные и нелинейные члены, то возможные значения для $s$ и $m$ будут следующие:

$$
\begin{array}{ll}
s=0 & s=1 \quad s=2 \\
m=-1 / 2, & m=0, \quad m=1 / 2, \ldots
\end{array}
$$

В дальнейшем исследуем асимптотическое разложение, используя первыми нз этих показателей $s=0, m=-1 / 2$. Вариант с $s=1, m=0$ подробно рассмотрен в [1]. Вариант с $s=2, m=1 / 2$ и последующие варианты не позволяют провести интегрирование по координате $\eta$ и поэтому с точки зрения двумерных расчетных моделей не представляют интереса.

Обозначим

$$
\pi=\varepsilon^{1 / 2}
$$

и введем новое безразмерное время по формуле

$$
\tau=\sum_{n=0}^{N} \tau_{n}(t)
$$

где

$$
\tau_{n}(t)=\sqrt{\frac{\mu}{\varrho}} \frac{1}{h} \pi^{n} t
$$

Производные по времени $t$ тогда выражаются через производные по $\tau_{n}-\frac{\partial}{\partial \tau_{n}}()=()_{; n}$ следующим образом:

$$
\frac{\partial}{\partial t}(\quad)=\sqrt{\frac{\mu}{\varrho}} \frac{1}{h} \sum_{n=0}^{N} \pi^{n}(\quad) ; n .
$$

Введены также следующие безразмерные величины:

$$
\begin{gathered}
u_{\alpha}=h v_{\alpha}, \quad u_{3}=\pi^{-1} h w \\
\sigma_{\alpha \beta}=\pi^{2} \mu \tau_{\alpha \beta}, \quad \sigma_{\alpha 3}=\mu \tau_{\alpha 33}, \quad \sigma_{33}=\pi^{-2} \mu \tau_{33} .
\end{gathered}
$$

Далее, пусть на цилиндрической граничной поверхности пластинки $C_{1}$ заданы внешние усилия

$$
P_{\alpha}=\pi^{2} \mu P_{\alpha}^{\prime}, \quad P_{3}=\pi^{3} \mu P_{3}^{\prime}
$$


и на -поверхности $C_{2}$ перемещения

$$
u_{\alpha}^{\prime}=h v_{\alpha}^{\prime}, \quad u_{3}^{\prime}=\pi^{-1} h w^{\prime} .
$$

Поверхности пластинки $\eta= \pm 1$ пусть будут нагружены нормальной нагрузкой

$$
p=\pi^{5} \mu p^{\prime}
$$

Если введенные новые координаты, перемещения и напряжения подставить в уравнения и соотношения геории упругости, то они примут следующий вид:

уравнения равновесия

$$
\begin{gathered}
A_{\gamma} \equiv \pi^{2}\left[\pi^{2} \tau_{\alpha \beta}\left(\delta_{\beta \gamma}+\pi^{2} v_{\gamma, \beta}\right)+\tau_{\alpha, 3} v_{\gamma, \gamma_{1}}\right]_{, \alpha}+ \\
+\pi^{-2}\left[\pi^{2} \tau_{3 \alpha}\left(\delta_{\alpha \gamma}+\pi^{2} v_{\gamma, \alpha}\right)+\tau_{33} v_{\gamma, \gamma_{1}}\right]_{, \gamma_{1}}-\sum_{n=0}^{N} \pi^{2 n} v_{\gamma, n n}=0 \\
A_{3} \equiv \pi\left[\pi^{4} \tau_{\alpha \beta} w_{, \beta}+\tau_{\alpha 3}\left(\tau+w_{, \gamma_{1}}\right)\right]_{, \alpha}+ \\
+\pi^{-3}\left[\pi^{4} \tau_{3 \alpha} w_{, \alpha}+\tau_{33}\left(\pi+w_{, \gamma_{1}}\right)\right]_{, \gamma}-\sum_{n=0}^{N} \pi^{2 n-1} w_{; n n}=0
\end{gathered}
$$

соотношения упругости

$$
\begin{aligned}
& \tau_{\alpha \beta}=\pi^{-2}\left(\delta_{\alpha \beta} k e_{i i}+2 e_{\alpha \beta}\right) \\
& \tau_{\alpha 3}=2 e_{\alpha 3} \\
& \tau_{33}=\pi^{2}\left[k e_{\alpha \alpha}+(2+k) e_{33}\right],
\end{aligned}
$$

где $\quad k=\lambda / \mu$

кинематические условия

$$
\begin{aligned}
& e_{\alpha \beta}=\frac{1}{2} \pi^{2}\left(v_{\alpha, \beta}+v_{\beta, \alpha}+w_{, \alpha} w_{, \beta}+\pi^{2} v_{\gamma, \alpha} v_{\gamma, \beta}\right) \\
& e_{\alpha 3}=\frac{1}{2}\left(v_{\alpha, \eta}+w_{, \alpha} w_{, \gamma}+\pi w_{, \alpha}+\pi^{2} v_{\gamma, \alpha} v_{\gamma, \gamma_{1}}\right) \\
& e_{33}=\frac{1}{2} \pi^{-2}\left(w_{, \gamma} w_{, \eta}+2 \pi w_{, \gamma}+\pi^{2} v_{\gamma, \gamma} v_{\gamma, \gamma}\right)
\end{aligned}
$$

условия на поверхностях $\eta= \pm 1$ пластинки

$$
\begin{aligned}
& B_{\beta} \equiv \tau_{3 \alpha}\left(\delta_{\alpha \beta}+\pi^{2} v_{\beta, \alpha}\right)+\pi^{-2} \tau_{33} v_{\beta, \gamma}=0 \\
& B_{3} \equiv \pi \tau_{3 \alpha} w_{, \alpha}+\pi^{-3} \tau_{33}\left(\pi+w_{, \gamma_{1}}\right) \mp \pi^{5} p^{\prime}=0
\end{aligned}
$$

условия на контурной поверхности $C_{1}$

$$
\begin{aligned}
& D_{\beta} \equiv T_{\alpha \beta} n_{\alpha}-P_{\beta}^{\prime}=0 \\
& D_{3} \equiv T_{\alpha 3} n_{\alpha}-P_{3}^{\prime}=0,
\end{aligned}
$$


где

$$
\begin{aligned}
& T_{\alpha \beta}=\pi^{2} \tau_{\alpha \gamma}\left(\delta_{\gamma \beta}+\pi^{2}{v_{\beta, \gamma}}\right) \\
& T_{\alpha \beta}=\pi^{3} \tau_{\alpha \beta} w_{, \beta}+\pi^{-1} \tau_{\alpha \beta}\left(\pi+\psi_{, \gamma}\right) .
\end{aligned}
$$

Здесь $n_{\alpha}$ - компоненты единичного нормального вектора контура пластинки.

Для рассматриваемой нелинейной задачи теории упругости можно сформулировать следующую вариационную задачу Гамильтона:

$$
\begin{gathered}
\delta F \equiv \int_{\tau_{0}}^{\tau_{1}}\left\{\pi^{-5} \int_{V}\left(\pi A_{\alpha} \delta v_{\alpha}+A_{3} \delta w\right) d V-\tau^{-5} \int_{S}\left(\pi B_{\alpha} \delta v_{\alpha}+B_{3} \delta w\right) d S-\right. \\
-\pi^{-3} \int_{C_{1}}\left(\pi D_{\alpha} \delta \tau_{\alpha}+D_{3} \delta w\right) d C-\pi^{-3} \int_{C_{2}}\left[\pi\left(v_{\alpha}-v_{\alpha}^{\prime}\right) n_{\beta} \delta T_{\alpha \beta}+\right. \\
\left.\left.+\left(w-w^{\prime}\right) n_{\alpha} \delta T_{\alpha 3}\right] d C\right\} d \tau=0
\end{gathered}
$$

Из этой вариационной задачи вытекают уравнения равновесия (1.12), статические краевые условия (1.15), (1.16) на поверхностях $\eta= \pm 1$, $C_{1}$ и геометрические краевые условия на поверхности $C_{2}$.

Предполагаем, что перемещения, напряжения и деформации, а также внешние нагрузки и заданные перемешения можно разложить в ряд по малому параметру $\pi$, т. е.

$$
Q=\pi^{g} \sum_{s=0}^{\infty} \pi^{s} \stackrel{s}{Q},
$$

где $Q$ обозначает любую из названных величин. Чтобы первый член разложения имел величину, отличную от нуля, $g$ надо брать следующим образом: для $v_{\alpha}, w, \tau_{i k}, e_{\alpha 3}, P_{\alpha}^{\prime}, P_{3}^{\prime}, p^{\prime}, v_{\beta}^{\prime}, w^{\prime} g=0$, для $e_{\alpha \beta} g=2$ и для $e_{33} \quad g=-2$.

Введем разложения (1.19) в функционал (1.18) и представим его в виде ряда

$$
\delta F=\pi^{-8} \sum_{s=0}^{\infty} \pi^{s} \delta \stackrel{s}{F}
$$

Первое и следуюшие приближения искомого напряженного состояния выведем из требования, чтобы вариация функционала $(1.18) \delta F$ равнялась нулю с заданной асимптотической точностью $\pi, \pi^{2}, \ldots$ Так как в функционале (1.18) все члены разложений перемещений $v_{\alpha}$, w будут рассмотрены как независимые варьируемые величины, то из условий $\delta \stackrel{n}{F}=0$ следует, что и $\delta \stackrel{r}{F}=0$ при всех $r<n$. Поэтому, если

$$
\delta \stackrel{n}{F}=0
$$

то условие $\delta F=0$ будет удовлетворено с асимптотической погрешностью $\pi^{n-7}$. Для первого приближения надо взять $n=8$, для второго $n=9$ и т. д. 
Из (1.21) вытекает следующая система уравнений движения:

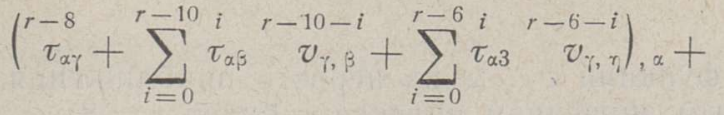

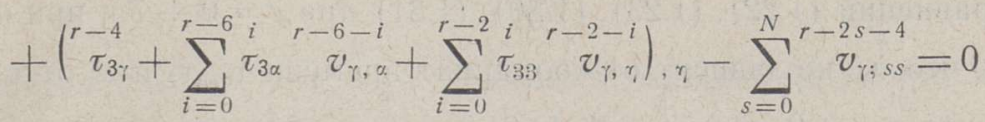

$$
\begin{aligned}
& \left(\sum_{i=0}^{r-8} \tau_{\alpha \beta}^{i} w_{, \beta}^{r-8-i}+{ }^{r-5} \tau_{\alpha, 3}+\sum_{i=0}^{r-4} \tau_{\alpha 3}^{r-4-i} w_{, \eta}\right)_{, \alpha}+
\end{aligned}
$$

$+\left(\sum_{i=0}^{r-4} \tau_{\alpha 3}^{i-4-i} w_{, \alpha}^{r-1}+\tau_{33}^{r-1}+\sum_{i=0}^{r} i_{33}^{i} \tau_{, \gamma_{i}}^{r-i}\right)_{, \eta}-\sum_{s=0}^{N} w_{i s s}^{r-2 s-2}=0, \quad(r=0,1, \ldots, n)$

где

$$
\begin{aligned}
& \tau_{\alpha \beta}^{s}=\delta_{\alpha \beta} k e_{\Upsilon \gamma}^{s}+2 e_{\alpha \beta}^{s}+\delta_{\alpha \beta} k \stackrel{s+2}{e_{33}} \\
& s_{\alpha 3}^{s}=2 e_{\alpha 3}^{s} \\
& { }_{\tau_{33}}^{s}=k{ }^{s-2} e_{\alpha \alpha}^{-2}+(2+k){ }^{s} e_{33}
\end{aligned}
$$

и

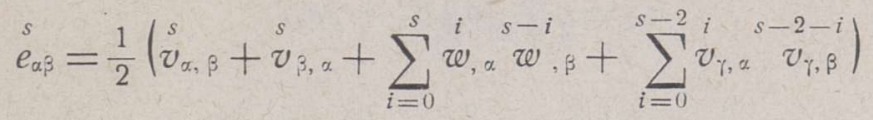

$$
\begin{aligned}
& e_{\alpha, 3}^{s}=\frac{1}{2}\left(\begin{array}{c}
s \\
v_{\alpha, \eta}
\end{array}+\sum_{i=0}^{s} w_{, \alpha}^{i} w_{, \eta}^{s-i}+w_{, \alpha}^{s-1}+\sum_{i=0}^{s-2} v_{\eta, \alpha}^{i} v_{\gamma, \eta}^{s-2-i}\right) \\
& e_{33}^{s}=\frac{1}{2}\left(\sum_{i=0}^{s} w_{, \eta}^{i} w_{, \eta}^{s-i}+2 w_{, \eta}^{s-1}+\sum_{i=0}^{s-2} v_{\eta, \eta}^{i} v_{\eta, \eta}^{s-2}\right)
\end{aligned}
$$

Так же получим из (1.18) следующие условия на поверхностях $\eta= \pm 1:$

$$
\begin{aligned}
& \tau_{3 \gamma}^{r-4}+\sum_{i=0}^{r-6} \tau_{3 \alpha}^{i} \tau_{\gamma, \alpha}^{r-6-i}+\sum_{i=0}^{r-2} \tau_{33}^{r-2-i} \tau_{\psi, \eta}=0 \\
& \sum_{i=0}^{r-4} \tau_{\alpha 3}^{r-4-i} w_{, \alpha}^{r-1}+\tau_{33}^{r}+\sum_{i=0}^{r} \tau_{33}^{i}{ }^{r-i} w_{, \eta} \mp \frac{1}{2}{ }^{r-8} p^{\prime}=0 . \quad(r=0,1, \ldots, n)
\end{aligned}
$$

В приведенных уравнениях и соотношениях считается, что $\stackrel{s}{Q}=0$ при $s<0$.

В следующем пункте проводится интегрирование системы (1.22)(1.31) по координате $\eta$. 


\section{2. Мембранная теория}

Определяем функции $v_{\gamma}$, $w$ из первого приближения, т. е считаем что максимальным значением индекса $r$ будет $r=8$.

Из уравнений $(1.22),(1.23),(1.30),(1.31)$ при $r=0 \div 7$ и при предположении, что $v_{f} v_{\gamma}$ не зависит от координат времени $\tau_{0}, \tau_{1}$ и $\underset{h}{s}$ от $\tau_{0}, \tau_{1}, \tau_{2}$. следует, что $\tau_{\alpha 3}=0$, если $f=0,1,2,3$, и $\tau_{33}=0$, если $h=0 \div 6$. Соотношения $(1.25),(1.26)$ теперь требуют, чтобы $e_{\alpha 3}=0$ если $j=0 \div 3$, $\stackrel{0}{e}_{33}=\stackrel{1}{e_{33}}=0$

$$
e_{33}^{2}=-\frac{k}{2+k}{ }^{0} e_{\alpha, \alpha}
$$

Далее из соотношений $(1.28),(1.29)$ вытекает, что

$$
v_{\gamma, \eta_{1}}=0, \quad \omega_{, r}=0
$$

и следовательно

$$
\stackrel{0}{v_{\gamma}}=\stackrel{0}{V_{\gamma}}\left(\xi_{\alpha,}, \tau_{2}\right), \quad \stackrel{0}{\omega}=\stackrel{0}{W}\left(\xi_{\alpha}, \tau_{3}\right) .
$$

Из соотношений $(1.24),(1.27)$ получается, что

$$
\tilde{\tau}_{\alpha \beta}=\frac{2 k}{2+k} \delta_{\alpha \beta} e_{\pi \gamma}^{0}+2 \stackrel{0}{2 e_{\alpha \beta}}
$$

ห

$$
\stackrel{0}{e_{\alpha \beta}}=\frac{1}{2}\left(\begin{array}{l}
0 \\
V_{\beta, \alpha}
\end{array}+\stackrel{0}{V_{\alpha, \beta}}+\stackrel{0}{W} \stackrel{0}{W} \underset{, \alpha}{W}\right)
$$

С учетом полученных результатов уравнения (1.22), (1.23), (1.30), (1.3і) при $r=8$ получают вид

$$
\begin{aligned}
& \tilde{\tau}_{\alpha \beta, \alpha}+\stackrel{4}{\tau_{3 \beta, \eta}}-\stackrel{0}{V} V_{\beta ; 22}=0
\end{aligned}
$$

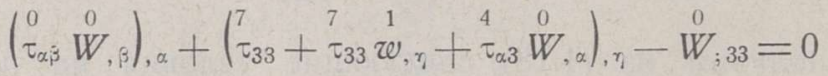

ห

$$
\begin{aligned}
& {\stackrel{4}{\tau \alpha}}=0
\end{aligned}
$$

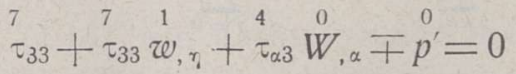

при $\eta= \pm 1$.

Проводя интегрирование по $\eta$ из уравнений $(2.6),(2.7)$ и соотношений $(2.8),(2.9)$, получаем следующие уравнения для определения функций $V_{\gamma}, W$ :

$$
{ }_{\tau}^{0}-\stackrel{0}{V}_{\beta ; 22}=0
$$




$$
\left.\left(\begin{array}{ll}
0 & 0 \\
\tau_{\alpha \beta} & W
\end{array}\right)_{, \beta}\right)_{, \alpha}-\stackrel{0}{W} ; ; 3=-\frac{1}{2} \stackrel{0}{p^{\prime}}
$$

и следующие значения для напряжений:

$$
\tau_{3 \alpha} \doteq 0
$$

Уравнения $(2.10),(2.11)$ - хорошо известные уравнения мембранной теории гибких пластинок. Отметим, что в этой теории $\stackrel{0}{V}$ з зависит от координаты времени $\tau_{2}$ и $\stackrel{0}{W}$ от $\tau_{3}$. Соответственно их изменения во времени характеризуются порядками $\varepsilon$ и $\varepsilon^{3 / 2}$.

Граничные условия теории получаются из двух последних интегралов функционала (1.18). Они имеют следующий вид:

статические условия на контуре $C_{1}$

$$
\begin{aligned}
& \tau_{\alpha \beta} n_{\beta}-\frac{1}{2} \int_{-1}^{+1} P_{\alpha}^{0} d \eta=0 \\
& \tau_{\alpha \beta} \stackrel{0}{W}, n_{\alpha}-\frac{1}{2} \int_{-1}^{+1} \stackrel{0}{P}_{3}^{\prime} d \eta=0
\end{aligned}
$$

геометрические условия на контуре $C_{2}$

$$
\begin{aligned}
& V_{\alpha}-\frac{1}{2} \int_{-1}^{+1} v_{\alpha}^{\prime} d \eta=0 \\
& \stackrel{0}{W}-\frac{1}{2} \int_{-1}^{+1} w^{\prime} d \eta=0 .
\end{aligned}
$$

Если выписать уравнения, определяющие второе приближение $\underset{1}{1} \frac{1}{v_{1}}, \underset{w}{,}$ и провести интегрирование по координате $\eta$, то получим, что $v_{\eta}$ и зависят от координаты $\eta$, т. е.

$$
\stackrel{1}{v_{\gamma}}=\stackrel{1}{V}\left(\xi_{\gamma}, \tau_{2}\right), \quad \stackrel{1}{w}=\stackrel{1}{W}\left(\xi_{\alpha}, \tau_{3}\right)
$$

и оказывается, что уравнения и граничные условия для функций $\stackrel{1}{V}, \stackrel{1}{W}$ имеют такой же вид, как соответствующие соотношения, получаемые из мембранной теории, если в последних фукнции $\stackrel{0}{V}, \stackrel{0}{W}$ заменить выражениями $\stackrel{0}{V}{ }_{\gamma}+\pi \stackrel{1}{V}, \stackrel{0}{W}+\pi \stackrel{1}{W}$. Следовательно, мембранная теория определяет напряженное состояние пластинки в случае $u_{\alpha} \sim h, u_{3} \sim \varepsilon^{-1 / 2} h$ с асимптотической погрешностью (в прйеняемой энергетической трактовке) $\varepsilon$. 
ЛИТ Е РА Т У Р A

1. А й н о л а Л. Я., Прикл. мех., № 8, 7-16 (1965)

Ннститут кибернетики

Академии наук Эстонской ССР

Псступила в редакцию

20/XII 1965

L. AINOLA

\section{ÖHUKESTE PLAATIDE DÜNAAMIKA ASÜMPTOOTILINE TEOORIA SUURTE LÄBIPAINETE KORRAL}

Geomeetriliselt mittelineaarse elastsusteooria võrrandid integreeritakse asümptootilisel meetodil siis, kui paigulused on suurusjärgus $u_{\alpha}-h \div \varepsilon^{1 / 2} h$ ja $u_{3} \backsim \varepsilon^{-1 / 2} h \div h(\varepsilon=h / L, 2 h-$ plaadi paksus, $L$ - plaadi laiust iseloomustav suurus $)$. Integreerimisel kasutatakse töös [ $\left.{ }^{1}\right]$ toodud metoodikat, mille kohaselt liikumise võrrandid ja ääretingimused tuletatakse tingimusest, et vastava funktsionaali variatsioon nõutud asümptootilise täpsuse korral võrdub nulliga. Selgub, et nimetatud suurusjärgus on plaadi paigutused määratavad membraaniteooriaga.

L. AINOLA

\section{THE ASYMPTOTIC DYNAMIC THEORY OF THIN PLATES BY LARGE DISPLACEMENTS}

The asymptotic integration of equations of the non-linear three-dimensional theory of elasticity in the case of thin plates is considered. It is assumed that the values of the displacements are of the orders $u_{\alpha} \sim h \div \varepsilon^{1 / 2} h, u_{3} \sim \varepsilon^{-1 / 2} h \div h\left(\varepsilon=\frac{h}{L}\right.$, where $2 h$ is the thickness, and $L$ - the characteristic length of the plate). The method of asymptotic integration which had been applied is the same as in the paper [ $\left.{ }^{1}\right]$ : the equations of motion and the natural boundary conditions are derived from the condition that the corresponding functional equals to zero within the required asymptotic accuracy. It has been established that the displacements can be determined by the membrane theory. 